\title{
Cadena de suministros y su impacto en la calidad del sector carrocero de la provincia de Tungurahua
}

\begin{abstract}
Supply Chain and its impact on the quality of the bodybuilding sector in the province of Tungurahua.
\end{abstract}

Bertha Andrea Almeida Chafla. ${ }^{1} \&$ Wilson Fernando Jiménez Castro. ${ }^{2}$

DOI: $\underline{\text { https://doi.org/10.33262/visionariodigital.v5i4.1874 }}$

\begin{abstract}
.
In Ecuador, there are a variety of activities that help the country's productive matrix, among them the bodywork production activities that have the greatest boom in the province of Tungurahua, so they always look for integral logistics that includes planning, organization and control of all the activities related to the reception of raw materials, transfer and storage of products, to satisfy the needs and requirements that the demand requires, in a fast, efficient way and with the lowest possible costs. Objectives: Identify how logistics influences to achieve a 4.0 supply chain, analyze the impact of quality on the competitiveness of the bodybuilding market. Methodology: The research design turns based on a quantitative approach to more than a descriptive method. The population was made up of 27 auto body companies from the province of Tungurahua, this information is mentioned based on the latest information from 2017, the questionnaire was made up of 14 items, the questionnaire to have the validity required for this research was previously approved by professionals on issues related to this research. Contributions: This article seeks to identify the origin of the different production processes through which a product goes through, as well as its subsequent logistics with its delivery to the final consumer, all this to the application of traceability. In addition to the recognition of the bodybuilding companies in the province of Tungurahua that implement the ISO 9001

Keywords: supply chain 4.0, ISO Standard Certification, logistics, competitiveness, traceability.

\footnotetext{
${ }^{1}$ Universidad Técnica de Ambato, Facultad de Ciencias Administrativas, Carrera de Marketing y Gestión de Negocios, Ambato, Ecuador. balmeida4855@uta.edu.ec. 0000-0001-5392-3480

${ }^{2}$ Universidad Técnica de Ambato, Facultad de Ciencias Administrativas, Carrera de Marketing y Gestión de Negocios, Ambato, Ecuador. wf.jimenez4855@uta.edu.ec. 0000-0002-8717-1501
} 


\section{Resumen.}

En Ecuador existen una variedad de actividades que ayudan a la matriz productiva del país entre ellas las actividades de producción de carrocerías que tiene mayor auge en la provincia de Tungurahua, por lo que siempre buscan una logística integral que incluya planificación, organización y control de todas las actividades conexas con la recepción de materia prima, traslado y almacenamiento de productos, para satisfacer las necesidades y los requerimientos que la demanda requiere, de una manera rápida, eficiente y con los menores costos posibles. Objetivos: Identificar cómo influye la logística para alcanzar a obtener una cadena de suministros 4.0, analizar el impacto de la calidad en la competitividad del mercado carrocero. Metodología: El diseño de la investigación gira en base a un enfoque cuantitativo a más de un método descriptivo. La población estuvo conformada por 27 empresas carroceras de la provincia de Tungurahua, esta información se la menciona en base a la última información del año 2017, el cuestionario estuvo conformado por 14 ítems, el cuestionario para que tenga la validez requerida para esta investigación fue previamente aprobado por profesionales en temas relacionados con esta investigación. Aportes: El presente artículo busca identificar el origen de los diferentes procesos productivos por los cuales atraviesa un producto, así como su posterior logística con su entrega al consumidor final todo esto a la aplicación de la trazabilidad. Además del reconocimiento a las empresas carroceras de la provincia de Tungurahua que implementan la certificación de la norma ISO 9001 para crear técnicas estandarizadas y de esta manera mejorar su calidad competitiva y los atributos de los productos que ofertan en el mercado.

Palabras claves: cadena de suministros 4.0, Certificación Norma ISO, logística, competitividad, trazabilidad.

\section{Introducción.}

La implementación de nuevas centrales de energía eléctrica, provoco cambios positivos en la matriz productiva, debido a que se abarataron los costos de energía que anualmente empleaban las empresas automotrices en el país, uno de los grandes sectores beneficiados es el sector de las carrocerías para auto buses, ya sean urbanos, interprovinciales o de turismo, por tanto, esta investigación es referente al sector carrocero, debido a que este sector industrial aporta significativamente al PIB del país, según estimaciones, se considera que el $13 \%$ proviene de esta área productiva, debido a que esta actividad económica conlleva la aplicación de ciertas áreas las cuales son: administrativa, financiera e industrial, para lo cual es necesario el contar con las herramientas con las cuales se pretende desarrollar controlar este tipo de procesos para que de esta manera la empresa emplee la totalidad de los recursos disponibles evitando perdidas que afecten su futuro. Hoy en día existen gremios mismos que son privados o públicos que ayudan al fortalecimiento del sector carrocero, mediante una asistencia técnica que se especializa en mejorar la calidad y la eficiencia en sus procesos productivos bajo el cumplimiento de normas como de reglamentos vigentes. 
Por lo antes expuesto el objetivo de la presente investigación es aplicar la herramienta de la trazabilidad para identificar el origen de los diferentes procesos productivos en el sector carrocero de la Provincia de Tungurahua es decir los procesos por los cuales atraviesa un producto, así como su posterior logística con su entrega al consumidor final.

También se puede acotar que el 31 de julio del 2007 el Ministerio de Industrias y Productividad aprobó la creación de la Cámara Nacional de Fabricantes y Carrocerías (CANFAC) la cual hace grandes proyectos de capacitación y calificación de soldadores para poder implementar la certificación de la Norma ISO 9001, fue así como en su segundo aniversario (julio 2009) CANFAC realizó la entrega oficial de la Certificación ISO 90012008 a las empresas que lograron conseguirlo como carrocerías VARMA, MIRAL, CEPEDA CÍA LTDA, CARROCERIAS PATRICIO CEPEDA Y SERMAN Cámara Nacional de Fabricantes y Carrocerías esto lo determina CANFAC (2012).

\section{Metodologia.}

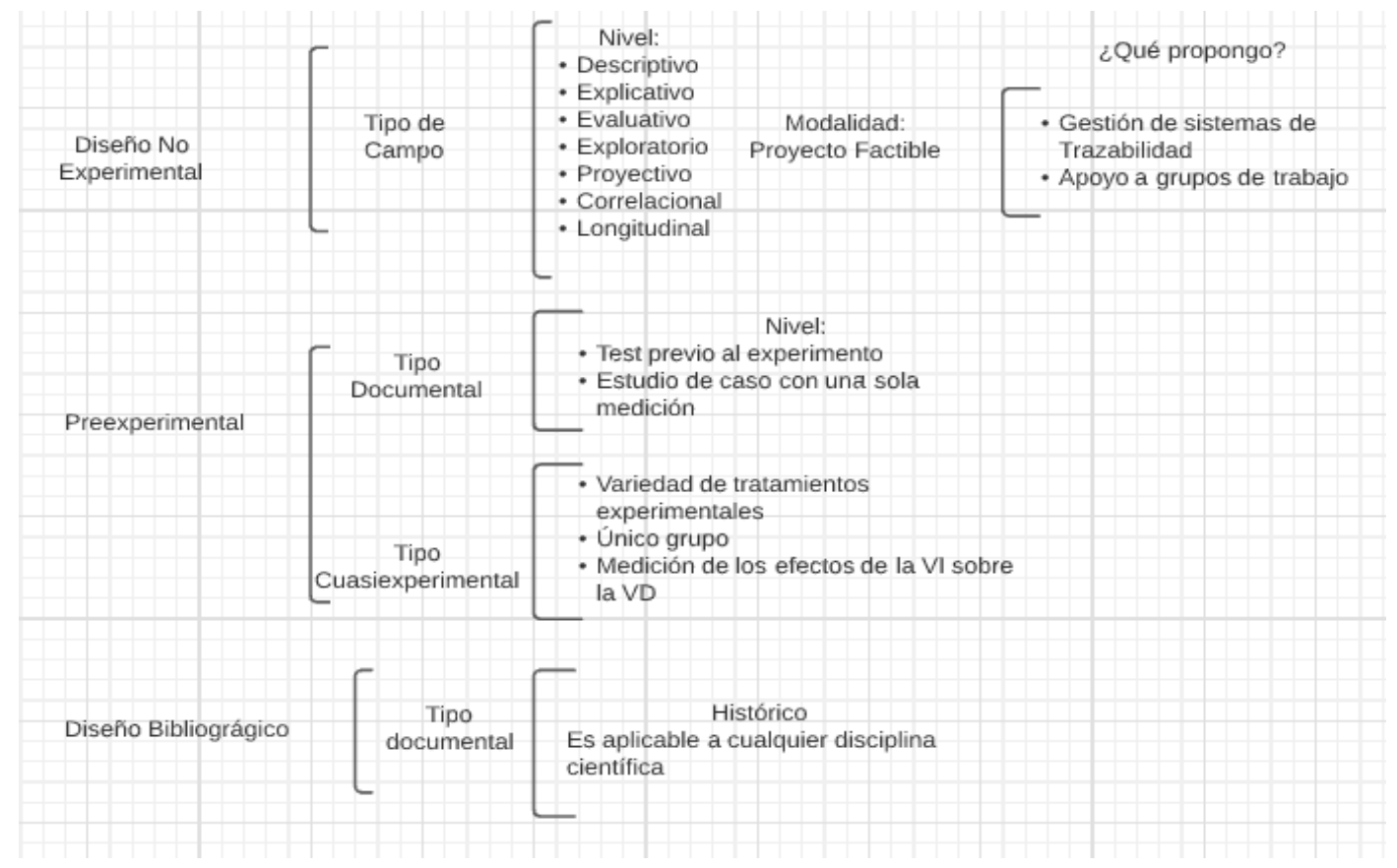

Ilustración 1. Diseño, tipo, nivel y modalidad de la investigación

Fuente: Elaboración propia

El uso de este diseño parte de la investigación de campo que en su mayoría es de nivel descriptivo debido a que se interpreta las realidades de un hecho del cual se elabora un análisis e interpretación de los datos que arroja la investigación, también se delimita futuras investigaciones por lo mismo se intenta proponer soluciones a posibles problemas que se den dentro de la investigación. Mide la relación entre dos variables (Cadena de suministros y calidad); las mismas que pueden ser longitudinales ya que pueden presentar cambios a futuro de las variables en estudio.

Además de poseer un amplio control de la formación de grupos de control el investigador puede manipular las variables antes de la observación. En el tipo bibliográfico se puede realizar un control de manera sistemática y profunda del material documental. 
La presente investigación está fundamentada con un método descriptivo enfocada en el método cualitativo, esto se debe debido a la naturaleza empleada en la elaboración de la herramienta de investigación, la cual utilizara la encuesta para poder recolectar la información necesaria con la cual se identificarán los datos necesarios para desarrollar los aspectos más importantes, de acuerdo con el autor Winston, (2019) afirma que este tipo de método consiste en mostrar el nivel de profundidad que puede determinarse, ya que por medio este proceso, lo que se hace es mostrar la realidad al investigador ya que este personaje se encuentra frente a frente con el problema que desea investigar (pág. 67), en el propósito principal de este documento es el de encontrar soluciones en base a la calidad de la cadena de suministros en el sector carrocero de la provincia de Tungurahua enfocados desde el abastecimiento, almacenamiento, inventarios, distribución (transporte) y logística. De esta manera poder identificar de una manera más detallada las fortalezas y debilidades con las que este sector cuenta.

En este caso, la herramienta de investigación seleccionada es la encuesta, según establece el autor Johnson (2018) confirma, que una de las herramientas más versátiles para poder recolectar datos de una manera ordenada y simple es la encuesta, la cual debe contar con un estudio previo en la cual se deben establecer las características que a su vez serán utilizadas para formular todas las preguntas necesarias para poder relacionar al investigador con el fenómeno que desea investigar, esto es de gran utilidad para desarrollar el proceso de investigación (pág. 54). El gran factor que desea buscar su nivel de incidencia es la trazabilidad se maneja en varios tipos según la cadena de suministros, por lo mismo es necesario realizar una distinción correcta de cada una de las etapas para que el sistema de trazabilidad funcione de manera correcta, ya que de ser el caso que se presente un error en alguna de las etapas, esto se puede ver reflejado en las etapas siguientes y realizar una toma de decisiones oportuna (Molina, 2014, pág. 56). Otro de los enfoques empleados es el método bibliográfico, según el autor Ortiz (2019), establece que el método bibliográfico se basa en fundamentar un texto científico mediante la búsqueda de información en fuentes bibliografías, estas pueden ser: libros, artículos científicos, artículos de revistas, etc, los cuales deben proveen del sustento científico necesario para desarrollar las variables descritas en cualquier trabajo de investigación. (pág. 44).

\section{Cadena de suministros}

Los autores Fontalvo et al., (2019) afirman que la cadena de suministros es un conjunto de procesos y flujos de recurso mismos que van desde la materia prima hasta el consumidor final, fuera del número de organizaciones que intervengan en este proceso formando así una gran red donde se maneja una comunicación asertiva y fluida; esto con el único objetivo de lograr la reducción e inclusive la eliminación de almacenamiento de inventario innecesario (pág. 145) . Para el criterio de los autores Cogollo y Criollo (2019) establecen que la cadena de suministros es un conjunto de características generales dentro de las cuales se establece una relación entre cliente - proveedores, los cuales están basados en la calidad desde el punto de vista de los clientes, ya que se puede mencionar 
que estos son los mejores jueces para toda empresa sin importar el giro de negocio (pág. 56).

Por otra parte, el autor Zuluaga et al., (2019) mencionan que la cadena de suministros o también conocida en inglés como Supply Chain Management, SCM) misma que consiste en alinear los procesos logísticos y sus actores que son: proveedores, distribuidores y clientes; para de esta manera lograr la satisfacción de necesidades y generar valor agregado a sus productos, reduciendo fallas y por ende realizando un uso eficiente de los recursos a utilizarse (pág. 45).

La innovación tecnológica ha realizado grandes cambios en la cadena de suministros ya que el sector carrocero de Tungurahua busca mayores espacios físicos para poder realizar un trabajo de fabricación y ensamble exitoso debido a que disponen de maquinaria de control numérico computarizado (CNC) y cabinas y hornos de pintura, esto hace que el porcentaje de esfuerzo físico de los colaboradores disminuya y por otro lado aumente su productividad. Cabe recalcar que los colaboradores actualizan sus conocimientos para ir de la mano con las implementaciones tecnológicas que este sector desarrolla por lo que varias empresas carroceras han obtenido la certificación ISO ya que mediante esta han logrado estandarizar la fabricación y ensamble reduciendo costos y mejorando la calidad, Cámara de Industrias de Tungurahua (CIT, 2016, pág. 65).

Cuando las organizaciones manejan una excelente logística en la cadena de suministros aumenta el valor de la organización ya que estas iniciativas dan impacto directo en el incremento de ingresos mediante el nivel de calidad en el servicio y la minimización de los costes en cuanto a la inexistencia de roturas de stock y las devoluciones. El incremento de los ingresos juntamente con la reducción de los costes mejora los beneficios antes de los intereses, amortizaciones y las depreciaciones. Algo que es notable cuando se maneja la cadena de suministros con la logística apropiada es que se reducen los activos circulantes debido a que la consecución de los niveles de inventarios es menor y el tiempo de cobro a los clientes también suele ser inferior, por otra parte, la reducción de activos fijos mejora la optimización de la red logística (Gómez, 2008).

Los componentes de la cadena de suministros son determinadas como un conjunto de tres o más entidades, misma que nace desde las materias primas extraídas del primer proveedor seguidos de los productos de venta y como último punto llega a los consumidores finales, para lo cual pasa por una serie de procesos que permiten ya sea de manera directa o indirecta la satisfacción del cliente Ocampo y Prada (2018).

La logística busca la rapidez y eficacia con la que se puede gestionar y organizar los sistemas de producción, pero todo esto va de la mano con la cadena de suministros para generar una oportuna coordinación tanto con los proveedores, clientes y juntamente con los canales de distribución; esto conlleva a una integración total de los elementos hablando así de una logística integral (Arbós, 2012, pág. 87) .

Para Callejas y Álvarez (2020) quienes mencionan que, la cadena de suministros puede ser reconocida como una red de empresas e individuos que poseen una mutua relación de 
dependencia, esto debido a la cantidad de información que se involucran entre todas las unidades de negocios interrelacionadas, las cuales están sujetas a las exigencias del consumidor final con respecto a los productos.

Por otra parte, para los autores Gutiérrez et al., (2018) mencionan que, la cadena de suministros es la coordinación sistemática y estratégica de los procesos dentro de una organización, los que desempeñan un papel muy importante en la satisfacción de los clientes y también de la generación de valor para todos los implicados ya que la cadena de suministros también puede ser considerada una red de empresas (pág. 78).

La cadena de suministro es considerada como un proceso integrado, donde la materia prima, después de un proceso de transformación, son presentados como productos terminados para finalmente ser entregados a los clientes mediante la distribución; por lo que la cadena de suministros se basa en cuatro eslabones complejos y gigantescos estos son: suministros, fabricación, distribución y consumidores si estos no son manejados y controlados de la manera correcta afectan en el rendimiento de las organizaciones Apolinario y Guevara (2021).

\section{Calidad}

Este término surgió para expresar lo que es un conjunto determinado de rasgos sobre un producto o servicio los mismos que se deben sustentar para de esta manera obtener un posicionamiento en la mente de los clientes mediante la satisfacción de sus necesidades (Navarrete et al., 2018, pág. 89).

Según Martínez (2019) menciona que, el término calidad ha traído innovaciones a través de los avances tecnológicos, debido a esto la mayoría de las organizaciones se someten a diario a la aplicación de mejoras continuas con dichos avances, para poder brindar ya sea un producto o servicio de calidad, al practicar estas estrategias se busca satisfacer las necesidades de los clientes (pág. 56) .

Los objetivos de la calidad han sido analizados de diferentes perspectivas, por una parte, se dice que busca la satisfacción total de los clientes bajo diferentes medios, y a su vez quiere lograr su máxima productividad lo que trae mayores utilidades, con el fin de alcanzar niveles óptimos de excelencia, por otro lado, busca la permanencia en el mercado que es lo que toda organización persigue independientemente del giro del negocio en que se desenvuelva (Carriel et al., 2018).

Para Hernández y Cano (2017) mencionan que, la calidad involucra varias actividades de planificación, organización y análisis de diversas áreas dentro de una organización, para que así los clientes perciban la calidad en los productos o servicios. Además, enfatizan que cuando se menciona el término de calidad se puede hablar de certificaciones, donde se consideran normas que deben cumplir algún tipo de especificaciones como los estándares de calidad que ya se encuentran predefinidos. Finalmente, dentro de la calidad también se analiza el desarrollo de la organización, de los recursos humanos y el compromiso de todos quienes conforman una organización. 
La Organización Internacional de Normalización (ISO 9000) define calidad como "el grado en que un conjunto de características inherentes cumple con los requisitos" (ISO, 2015). Cuando se habla de calidad es referirse a procedimientos que son efectuados en una organización con el fin de obtener resultados previstos, basados en el principio de calidad total esto quiere decir con una disminución de costos, tiempo y errores para así obtener excelentes resultados los que se ven reflejados en la competitividad de las organizaciones (Carrión mm cv., 2017).

El autor Casas (2018) menciona que, el término de calidad ha ido adquiriendo constantes cambios a través del tiempo, esto se debe a que los clientes (consumidores) están vinculados con la calidad desde que el hombre empezó con la creación de productos como alimentos, vestimenta, transporte, etc.

Según los autores Guzmán et al.,(2017) mencionan que, la calidad tiene algunas etapas las cuales demuestran su evolución entre ellas tenemos: la primera, es conocida como control de calidad la que se refiere al muestreo o a un control estadístico de los outputs o salidas mayormente reconocidas como productos; la segunda denominada como el aseguramiento de la calidad y de prevención, que hace referencia a un conjunto de acciones planificadas las mismas que son necesarias para proporcionar la confianza a un producto o servicio determinado los cuales cumplirán con requisitos de calidad. Como última de las etapas, la gestión de la calidad total, la que se refiere al uso de un sistema determinado que es empleado en la organización con el objetivo de buscar la satisfacción del cliente interno y externo, el mejoramiento continuo y la competitividad (pág. 67).

\section{Trazabilidad ascendente}

Esto hace referencia hacia atrás en el tiempo la misma que permite conocer cuáles son los materiales que entran a la empresa desde un lote e inclusive el consumo preferente y conocer además el nombre de los proveedores.

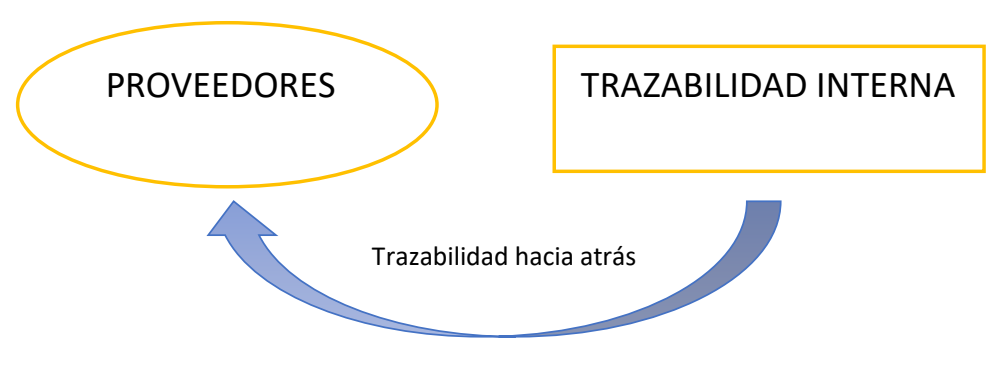

Ilustración 2: Trazabilidad ascendente
Fuente: Elaboración propia

\section{Trazabilidad interna}

Esto hace mención del hecho de realizar un seguimiento a todos los pasos que se dan dentro de la empresa, básicamente es vincular los productos que entran, con los que se 
distribuyen a los clientes. Debido a esto se debe controlar el historial desde el almacenaje, hasta que los productos son procesados dentro de la empresa.

\section{Trazabilidad descendente}

También conocida como trazabilidad hacia delante en el tiempo esta se basa en cambio a los productos que salen de la empresa y por contrario de la anterior esta desea conocer a sus distintos clientes.

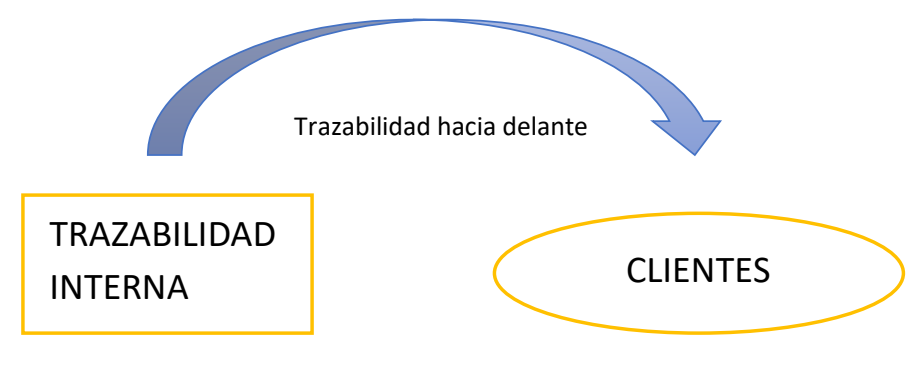

\section{Ilustración 3: Trazabilidad descendente} Fuente: Elaboración propia

Por otra parte, a modo de un resumen en la siguiente imagen se puede visualizar los tres tipos de trazabilidad.

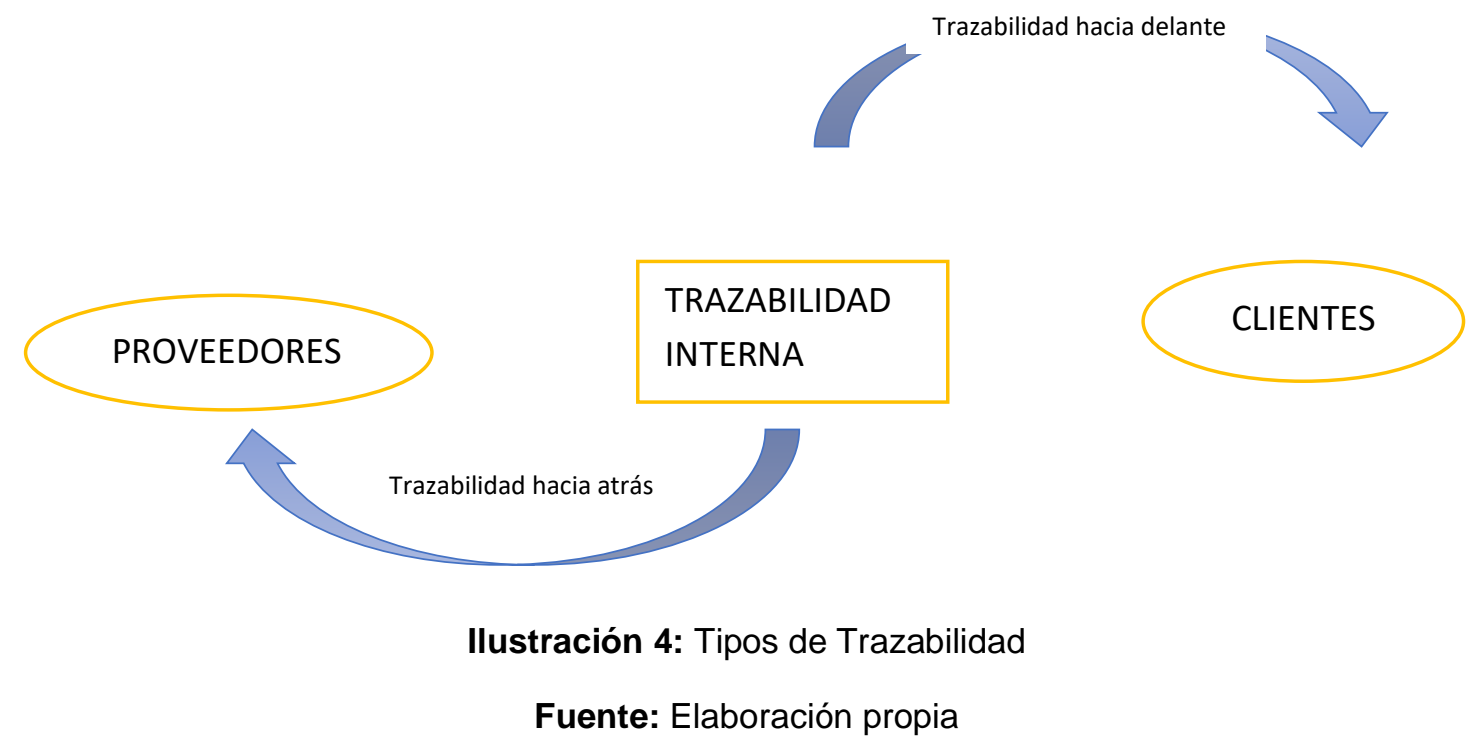

Por otra parte, el autor Johnson (2018) afirma que, la trazabilidad está basado en el Sistema de Gestión de Calidad ya que todas las empresas del sector carrocero andan tras la búsqueda constante de obtener una Certificación y Normalización en la norma ISO 9001:2015 la misma que promueve de una manera diferente la gestión de calidad en una organización, ya que a partir de los requisitos tradicionales de orientación a los clientes y los procesos, relaciones con clientes, empleados, proveedores, sociedad etc.; y la mejora de la calidad en todos los ámbitos de la organización (pág. 8). Es por ello por lo que la 
trazabilidad controla e identifica la entrada a las empresas tanto internos como externos para poder dar soluciones oportunas y ágiles a posibles reclamos.

\section{Gestión del Sistema de Trazabilidad}

Según considera los autores Callejas y Álvarez (2020)Este sistema cuenta con algunos requerimientos como el seguimiento que es el poder acceder a toda la información de la línea de fabricación y ensamblaje en donde se conoce la condición de cada puesto desde que empieza la jornada laboral. Por otra parte, también se hace uso de las consultas históricas que se basa en algunos criterios como es el detalle de las operaciones cumplidas, datos y resultados obtenidos además del detalle de la producción y su resultado final (pág. 167). El objetivo de manejar un control de trazabilidad es apoyar a los flujos de trabajo en cada iteración, es decir pueden tomar decisiones en base al desarrollo y del impacto de cambio, por lo mismo se podrá realizar negociaciones de éxito y sobre todo oportunas.

La trazabilidad según el autor Ortiz (2019) es considerada la capacidad de seguir un proceso preestablecido sobre la trayectoria de un producto a lo largo de la cadena de suministro, el gran desafío de la trazabilidad en la cadena de suministros es el intercambio de información ya que posee un formato estandarizado entre los actores que intervienen en la cadena. Esta información es intercambiada de una forma precisa, además que hace un énfasis en las alianzas estratégicas entre los fabricantes y los proveedores. Para garantizar que una cadena de suministros sea exitosa debe existir una asimetría en la información (pág. 146).

\section{Resultados}

Tabla 1

Porcentaje de importación de la materia prima y lugar de procedencia.

\begin{tabular}{cccc}
\hline \multicolumn{4}{c}{ Resumen de procesamiento de casos } \\
\hline \multirow{3}{*}{ Casos } & \multicolumn{3}{c}{$\%$} \\
\cline { 2 - 4 } & Válido & 27 & 100,0 \\
\cline { 2 - 4 } & Excluido & 0 &, 0 \\
\cline { 2 - 4 } & Total & 27 & 100,0 \\
\hline
\end{tabular}

Fuente: Elaboración propia

Esta tabla es una representación del procesamiento de todos los casos recopilados durante la aplicación de la herramienta de investigación, en la cual se puede apreciar que esta investigación cuenta con una muestra de 27 empresas manufactureras de autobuses.

Tabla 2

Nivel de Confiabilidad

\begin{tabular}{rlr}
\hline & Estadísticas de fiabilidad & \\
\hline $\begin{array}{c}\text { Alfa de } \\
\text { Cronbach }\end{array}$ & $\begin{array}{c}\text { Alfa de Cronbach basada en } \\
\text { elementos estandarizados }\end{array}$ & $\begin{array}{c}\mathrm{N} \mathrm{de} \\
\text { elementos }\end{array}$ \\
\hline, 915 & \multicolumn{2}{c}{12} \\
\hline \multicolumn{4}{c}{ Fuente: Elaboración propia }
\end{tabular}


Es necesario realizar este análisis de fiabilidad en SPSS para poder garantizar la validez y la precisión del análisis estadístico, basados en esta herramienta se aprecia en la tabla 2, que el alfa de Cronbach de 0,91 lo que quiere decir que los ítems de la encuestan están aportando al factor de esta investigación, creando confianza en los resultados que se obtuvo.

Tabla 3

Precepciones de la Trazabilidad de una empresa.

\begin{tabular}{ccc}
\hline & $\begin{array}{l}\text { ¿Conoce usted } \\
\text { sobre el significado } \\
\text { de Trazabilidad? }\end{array}$ & $\begin{array}{l}\text { ¿Cuenta la } \\
\text { empresa con un } \\
\text { modelo de } \\
\text { medición de } \\
\text { Trazabilidad? }\end{array}$ \\
\hline SI & 24 & 24 \\
\hline NO & 3 & 3 \\
\hline TOTAL & 27 & 27 \\
\hline \multicolumn{3}{c}{ Fuente: Elaboración propia }
\end{tabular}

De acuerdo, con los datos obtenidos el sector carrocero tiene conocimiento de la trazabilidad misma que hace referencia a todos los procedimientos por los que se somete un producto, desde sus inicios, de cómo es su evolución en cada etapa, por lo que se aprecia que, de las 27 empresas carroceras, 24 conocen sobre el significado de trazabilidad y solo 3 empresas desconocen del tema. Además, según datos arrojados, las empresas carroceras de la provincia de Tungurahua cuentan con un modelo de trazabilidad, ya que 24 de las empresas trabajan con un modelo de trazabilidad, mientras que 3 empresas no la aplican basadas en el mismo desconocimiento.

Tabla 4

Materia prima importada

\begin{tabular}{rccc}
\hline \multicolumn{4}{c}{ ¿Qué porcentaje de la materia prima es importada y de qué país } \\
proviene?
\end{tabular}

Fuente: Elaboración propia

Las empresas carroceras de la provincia de Tungurahua se manejan con un porcentaje de $71 \%$ de materia prima, por lo que mediante los resultados arrojados 16 empresas usan materia prima importada; 10 de las empresas se manejan con $61 \%-70 \%$ con materia prima importada, mientras que solo 1 empresa se maneja con un porcentaje bajo entre el 51 y $60 \%$ de materia prima importada. Además de Brasil proviene la mayor parte de materia prima. 
Tabla 5

Materia Prima Nacional Empleada

\begin{tabular}{lcc}
\hline $\begin{array}{c}\text { ¿Cuál es el porcentaje de materia prima nacional } \\
\text { utilizada en la cadena de suministros? }\end{array}$ \\
\hline $\mathbf{1 1 \%}-\mathbf{2 0 \%}$ & 10 & 37,0 \\
\hline $\mathbf{2 1 \%}-\mathbf{3 0 \%}$ & 16 & 59,3 \\
\hline $\mathbf{3 1 \%}-\mathbf{4 0 \%}$ & 1 & 3,7 \\
\hline Total & 27 & 100,0 \\
\hline
\end{tabular}

Fuente: Elaboración propia

Se observa que el porcentaje de 21 a $30 \%$ de materia prima es el rango con el que más se manejan las empresas carroceras ya que un $59,3 \%$ utilizan este porcentaje de materia nacional en su cadena de suministros.

Tabla 6

Estadísticas de los Ítems Restantes

\begin{tabular}{lcc}
\hline & Muy importante & Importante \\
\hline $\begin{array}{l}\text { ¿Qué tan importante es para usted la } \\
\text { mano de obra calificada en la calidad de }\end{array}$ & 44,4 & 55,6 \\
sus productos? & & \\
\hline $\begin{array}{l}\text { ¿Considera usted determinante la } \\
\text { aplicación de las TIC's en el }\end{array}$ & 37,0 & 63,0 \\
funcionamiento de la cadena de & & \\
suministros? & & \\
\hline $\begin{array}{l}\text { ¿Es para usted fundamental el detectar } \\
\text { las ineficiencias de la cadena de } \\
\text { suministros le ayuda a realizar una } \\
\text { oportuna toma de decisiones? }\end{array}$ & 48,1 & \\
\hline $\begin{array}{l}\text { ¿Usted Considera relevante el plazo } \\
\text { acordado para la entrega de pedidos? }\end{array}$ & 44,4 & 35,6 \\
\hline $\begin{array}{l}\text { ¿Es esencial para usted medir la relación } \\
\text { con los clientes en base a la calidad de } \\
\text { los productos? }\end{array}$ & 59,3 & \\
\hline $\begin{array}{l}\text { ¿Considera usted importante el conocer } \\
\text { la calidad de los productos frente a los } \\
\text { requerimientos del mercado? }\end{array}$ & 63,0 & 37,0 \\
\hline
\end{tabular}

Fuente: Elaboración propia

Como se aprecia, la mano de obra calificada es de gran importancia para la calidad de los productos con un 55,6\%; además la aplicación de las TIC's también lo consideran importante con un $63 \%$ en la cadena de suministros; considerando que es fundamental el detectar las ineficiencias de la cadena de suministros con un 51,9\% para que puedan realizar una toma de decisiones oportuna; otro factor considerado importante son los plazos acordados para la entrega de pedidos con un 55,6\%; por lo que también es muy importante que se pueda realizar una medición de la calidad con relación a los clientes 
con un 59,3\% esto debido a que sus perspectivas deben ser satisfechas en su totalidad por lo que la calidad está inmersa en esta medición,; finalmente para lograr el ítem anterior es muy importante conocer la calidad de los productos con un $63 \%$ frente a los requerimientos del mercado.

\section{Conclusiones}

- Como primera instancia tenemos que la trazabilidad es conocida y manejada por las empresas carroceras de la provincia de Tungurahua para un correcto manejo de los procesos y de esta manera la cadena de suministros tenga un control adecuado de todo lo que implica la realización de sus productos, para que estos al final de todos los procesos por los que atraviesa sean de calidad y cumplan con las normas y reglamentos vigentes para de esta manera lograr la satisfacción de las necesidades de los clientes.

- De igual manera, se considera que la trazabilidad aporta de una manera significativa ya que cabe recalcar que no es solo seguir los procesos por los que pasa un producto sino también de certificaciones de calidad que las empresas pueden obtener, lo que causa que las empresas quieran evitar los errores en los procesos de fabricación, ya que la obtención de estos certificados aporta en las empresas un estatus y un reconocimiento por parte de su competencia y sus clientes.

- Es así como se puede mencionar que las empresas carroceras de la provincia de Tungurahua consideran que la mano de obra de calidad es de vital importancia para la calidad de sus productos, por lo que las empresas están preocupadas de aquello y están en constante innovación de sus tecnologías para lo cual sus trabajadores deben saber cómo manejarlas para que la calidad de sus productos siga adelante y para ello el proceso de la cadena de suministros no debe tener ningún error.

- Finalmente, para que su cadena de suministros cumpla con la calidad requerida la mayoría de las empresas carroceras de la provincia de Tungurahua adquieren materia prima importada, a pesar de que los costos sean altos lo que importa es brindar productos de calidad a sus distinguidos clientes.

\section{Referencias bibliográficas.}

Apolinario, R., \& Guevara, D. (2021). El efecto mediador de la capacidad ejecutiva para la innovación entre la gestión del conocimiento. Información Tecnológica,32(1), 151-168. doi:http://dx.doi.org/10.4067/S0718-07642021000100151

Arbós, L. C. (2012). Logística. Gestión de la cadena de suministros. Ediciones Díaz de Santos.

Callejas, L., \& Álvarez, K. (2020). Trazabilidad en la cadena de suministro alimentaria:

Un estudio bibliométrico. Escome,11(2), 277-297. Obtenido de https://www.escolme.edu.co/revista/index.php/cies/article/view/309/335 
Cámara Nacional de Fabricantes de Carrocerias [CANFAC]. (2012). Promiviendo el desarrollo de la industria carrtocera.

https://es.calameo.com/books/004840217be1816e944c5

Carriel, R., Barros, C., \& Fernández, F. (2018). Sistema de gestión y control de la calidad: Norma ISO 9001:2015. Revista Científica Mundo de la Investigación y el Conocimiento,2(1), 625-644. doi:10.26820/recimundo/2.1.2018.625-644

Carrión, H., Mendoza, M., \& Vera, C. (2017). Importancia de la auditoría interna para el perfeccionamiento de los niveles eficiencia y calidad en las empresas. Dominio de las Ciencias,3(2), 1-13. doi:http://dx.doi.org/10.23857/dom.cien.pocaip.2017.3.2.esp.40-65

Casas, B. d. (2018). La ISO 9001 y la Administración de la Calidad Total en las Empresas Peruanas. Universidad \& $\quad$ Empresa,20(35), $\quad 1-32$. doi:http://dx.doi.org/10.12804/revistas.urosario.edu.co/empresa/a.6056

Cámara de Industrias de Tungurahua [CIT]. (2016, 24 de febrero). 70\% de la Industria Carrocera es Tungurahuense.

https://camaradeindustriasdetungurahua.wordpress.com/2016/02/24/70-de-laindustria-carrocera-es-tungurahuense

Cogollo, J., \& Correa, A. (2017). La gestión de la calidad en cadenas de suministro: Desarrollos y tendencias. Espacios, 38(37), 1-22. Obtenido de https://www.revistaespacios.com/a17v38n37/a17v38n37p16.pdf

Cogollo, J., \& Ruíz, C. (2019). Prácticas de responsabilidad sostenible de cadenas de suministro: Revisión y propuesta. Revista Venezolana de Gerencia, 24(87), 1-14. Obtenido de https://www.redalyc.org/articulo.oa?id=29060499004

Fantalvo, T., Hoz, E. d., \& Mendoza, A. (2019). Los Procesos Logísticos y La Administración de la Cadena de Suministro. Saber, Ciencia Y Libertad, 14(2), 102-112. doi:https://doi.org/10.18041/2382-3240/saber.2019v14n2.5880

Gómez, M. G. (2008). Cuantificación y generación de valor en la cadena de suministro extendida. España: Del Blanco Editores.

Gutiérrez, A., Rodríguez, C., \& Hernández, A. (2018). Factores críticos de éxito para la implementación de Business Process Management (BPM): estudio de caso sobre la cadena de suministro de una empresa del sector de la floricultura. Revista EAN, 85-108. doi:https://doi.org/10.21158/01208160.n0.2018.2019

Guzmán, B., Tarapuez, E., \& Parra, R. (2017). Estrategia y calidad en empresas colombianas de servicios. Revista Venezolana de Gerencia,22(80), 1-15. Obtenido de https://www.redalyc.org/articulo.oa?id=29055967004 
Hernández, C., \& Cano, M. (2017). La importancia del benchmarking como herramienta para incrementar la calidad en el servicio en las organizaciones. DSPACE, 1-12. Obtenido de http://148.202.167.116:8080/xmlui/bitstream/handle/123456789/3577/Importanc ia_benchmarking_como_herramienta.pdf?sequence $=1 \&$ isAllowed $=y$

Organización Internacional de Normalización [ISO]. (2015). Sistemas de Gestión de Calidad.

https://www.iso.org/obp/ui/\#iso:std:iso:9001:ed-5:v1:es

Johnson, R. (2018). "Exploratory investigation" en Asociation for Environmental and Outdoor Education. Boston: fluidsurveys.

Martínez, O. (2019). Logística integral y calidad total, filosofía de gestión organizacional orientadas al cliente. Interdisciplinaria Koinonía,4(7), 202-232. Obtenido de https://dialnet.unirioja.es/servlet/articulo?codigo=7062704

Molina, F. T. (2014). Preparación de pedidos, recepción, almacenaje y distribución de productos. España: Ideas Propias Editorial.

Navarrete, M., Pilamunga, E., \& Ramos, N. (2018). Herramientas de calidad total y la competitividad: caso sector carrocero de la provincia de Tungurahua. Visionario Digital,2(4), 50-67. doi:https://doi.org/10.33262/visionariodigital.v2i4.99

Ocampo, P., \& Prada, R. (2018). Orientación a la cadena de suministro y su relación con la gestión de proveedores una revisión bibliográfica. Universidad EAN, 1-9. Obtenido de https://www.researchgate.net/profile/Ricardo-Prada2/publication/322808122_Orientacion_a_la_cadena_de_suministro_y_su_relaci on_con_los_proveedores_Una_revision_bibliografica/links/5a710407aca272e42 5ed391c/Orientacion-a-la-cadena-de-suministro-y-su-relacio

Ortiz, J. (2019). Investigacion exploratoria: tipos, metodologia y ejemplos,. Mexico: Lifeder.

Winston, K. (2019). Exploratory research: definition, methods and examples. New York: study.com.

Zuluaga, A., Ceballos, N., \& Palacio, D. (2019). Gestión de la cadena de suministros y productividad en la literaturacientífica. I + D Revista de Investigaciones, 14(2), 112. doi:https://doi.org/10.33304/revinv.v14n2-2019004

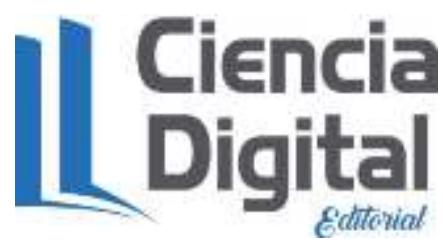




\section{PARA CITAR EL ARTÍCULO INDEXADO.}

Almeida Chafla, B. A., \& Jiménez Castro, W. F. (2021). Cadena de suministros y su impacto en la calidad del sector carrocero de la provincia de Tungurahua. Visionario Digital, 5(4), 6-20. https://doi.org/10.33262/visionariodigital.v5i4.1874

\section{Ciencia}

El artículo que se publica es de exclusiva responsabilidad de los autores y no necesariamente reflejan el pensamiento de la Revista Visionario Digital.

El artículo queda en propiedad de la revista y, por tanto, su publicación parcial y/o total en otro medio tiene que ser autorizado por el director de la Revista Visionario Digital.
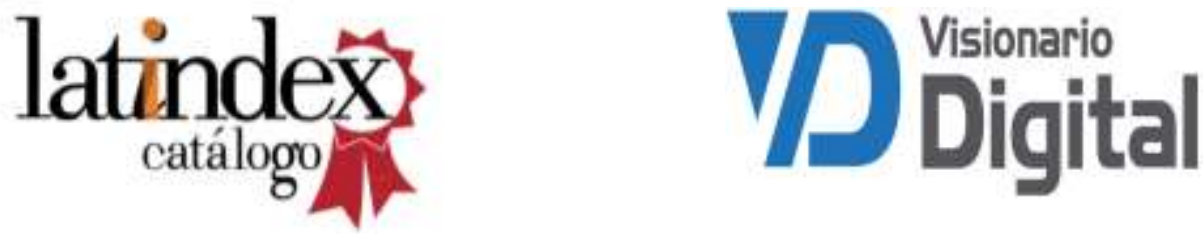